\title{
Collaborative Agent Learning Using Neurocomputing
}

\author{
Saulat Farooque ${ }^{1}$, Ajith Abraham ${ }^{2}$ and Lakhmi Jain ${ }^{1}$ \\ ${ }^{1}$ School of Electrical and Information Engineering, \\ University of South Australia, Adelaide, Australia \\ saulat.farooque@tenix.com, lakhmi.jain@unisa.edu.au \\ ${ }^{2}$ Department of Computer Science, Oklahoma State University, USA \\ ajith.abrahameieee.org
}

\begin{abstract}
In this paper we investigate techniques to train an agent to accomplish certain tasks. Artificial Neural Networks will be the technique used to the train the agent. This paper will investigate the use of Generalised Regression Neural Network (GRNN) to create and train agents capable of detecting face images. This agent would make up the 'Detection Agent' in an architecture comprising of several different agents that collaborate together to detect and then recognise certain images. The overall agent architecture will operate as an Automatic Target Recognition' (ATR) system. The architecture of ATR system is presented in this paper and it is shown how the detection agent fits into the overall system. Experiments and results using detection agent is also presented.
\end{abstract}

\section{Introduction}

The concept of software identities that have the ability (or intelligence) to perform some of the tasks that humans perform has great potential for numerous reasons. Firstly, intelligent agents could be used to provide humans suggestions or make decisions for them in response to a query. Secondly, intelligent agents could be deployed in dangerous situations to make decisions on behalf of humans. Lastly, intelligent agents could also be utilised to perform tasks that are too difficult for humans such as complex computation or quickly responding to certain stimuli that humans may be too slow for. Such intelligent agents have great potential in many diverse industries ranging from commerce to defence.

Programming languages often employ controls such as the "if" statement, which acts as a trigger for an event to occur. This represents a form of intelligence, where the software reacts to a certain stimulus. However, this is a static form of intelligence, which never changes with different situations. Dynamic intelligence is more similar to what humans encounter, i.e. having the ability to adapt to different types of situations. This is mainly a result of humans having the ability to learn and recognise different situations [6]. Likewise software can also be written to adapt, recognise and learn from pervious experiences [5]. 
Neurocomputing is one such method that employs the process of learning to mimic the learning process in humans. In this research study we deployed neural networks to train agents to accomplish certain tasks. The role of the agents developed for the case study will be to operate together as an 'Automatic Target Recognition' (ATR) system which should be able to collaborate with each other to detect the presence of faces within an image. Such a technology will exploit the ability of intelligent agents to learn from previous experiences (to identify face images) and then combine this knowledge with image processing techniques to extract and identify faces from within a regular image containing other details. This investigation will explore the ability of intelligent agents to learn knowledge and to accomplish a task by collaborating with other agents within the system. In Section 2 we present the architecture of ATR system followed by experiment results in Section 3. Some conclusions are also provided towards the end.

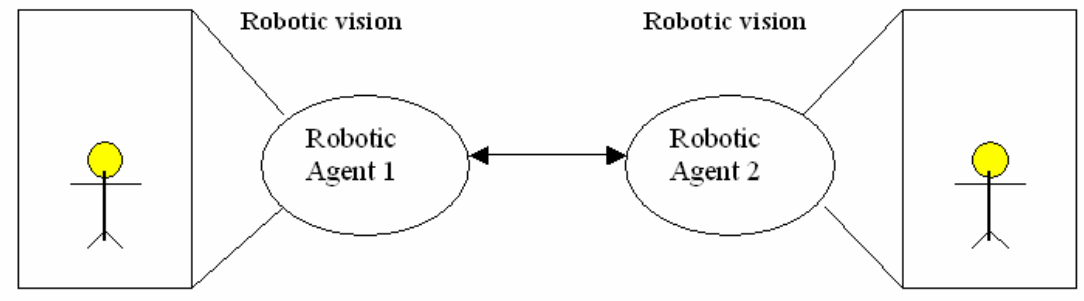

Fig.1. Robotic Agents

\section{Architecture of ATR}

It has been suggested that intelligent learning agents could be used in robots for defence purposes. Such robots could be thrown into buildings and then asked to travel though corridors visualising their environment. The aim of these robots would be to seek out objects (ie humans, bombs) and inform other robots or humans about knowledge gained. Figure 1 below describes how intelligent learning agents embedded in robots could seek out humans and then share information between another agents. Since the aim of the project is to be able to detect objects (whether it be human, animal or non-living), several different types of sensors could be within the Agents. Heat or motion sensors can be embedded into agents to detect the presence of humans and animals. However these sensors may fail if the object is stationary or non-living. Imaging sensors could be a good alternative in such scenarios. A complete and comprehensive intelligent agent could employ all the three sensors (heat, motion and image sensors) to gather information and exchange between other Agents to establish certain facts. However for the purpose of this investigation only knowledge gained from imaging sensors is examined.

Before any knowledge can be exchanged between robots an image scanned by a robot must be processed and a decision must be made whether a human is present in the image. This is where intelligence and learning is introduced to these robots. The overall aim of this project is to investigate such a concept. The concept of agents learning from a knowledge base can be tested on an ATR system where agents 
can automatically detect and recognise images. Such an ATR system could be created to investigate collaborative agent learning. Architecture for the development of the ATR system described above could comprise of two different stages: The Learning Stage and the Detection Stage. The learning stage would teach an agent what a person looks like. This process would make up the knowledge base for the ATR system. This could be done using ANN and would utilise a series of face images. The entry point into the ATR System would be via the Detection Stage. This would take images that a robot scans, and tries to detect if there are targets or images of interest within the picture. If possible targets are detected, these images are clipped and a Region Of Interest (ROI) is determined. This information is then passed onto the recognition agent. The recognition agent would refine the search and would determine whether the selected images are of the required targets (people's faces). Once it has been established that a person's face has been detected this information could then be shared between any other robot in the system. The learning stage could be developed as a learning agent that distributes information to the detection Stage and the recognition Stage. Figure 2 describes how the ATR system would work with the two different stages.

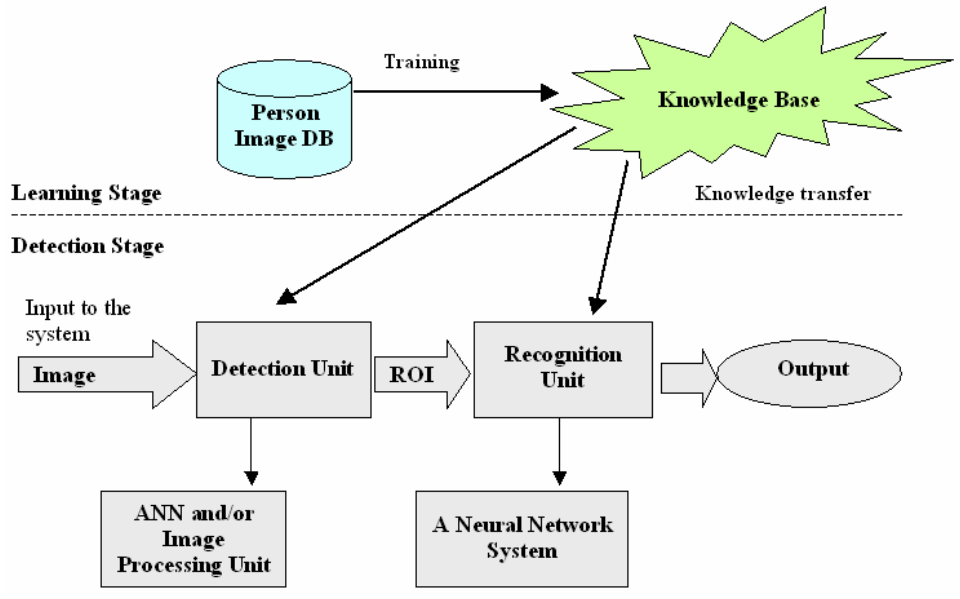

Fig.2. Architecture of ATR

The learning stage comprises of the single learning agent and the detection stage comprises of two distinct agents: the detection agent and the recognition agent. This agent architecture would operate by having the learning agent constantly training the detection and recognition Agents. This would also comprise of constantly updating the database of face images for training purposes. The detection and recognition agents would have a single role of detecting possible regions of interest (detection agent) and then recognising specific images from these regions of interest (recognition agent). This paper is more focused on the development of detection agents.

\subsection{Functioning of Detection Agent}


The role of the detection agent is to detect its target image, whether it be of a person's face as in Figure 3 (a), or any other image that the agent wishes to detect. The detection agent is there to simply detect the presence of the target image and pass all the probable candidates off to the recognition Agent. We modeled the detection agent using a two layer Generalised Regression Neural Network (GRNN). To increase the likelihood of the neural network detecting the target image, the target image is divided into tiles during the training process. The tiles created on the target image form non-overlapping grids, with each region of the grid being made up of binary values of 1 and 0 . The training is then performed on these tiles rather than the target image itself. This ensures a higher success rate if incomplete or distorted images are presented to the network once it has been trained. The number of output neurons for such a network is dependent on the number of target images (in this case just one). Each tile of the target image is assigned the same unique output indicating that it belongs to that face image. The horizontal rows of pixels in each tile are concatenated to create the network training vector. The first layer has the same number of neurons as there are inputs (for a face image tiled as a $15 \times 15$ grid, there would be 225 inputs). Each input is then subjected to a weight, derived from the transpose of the input vector of training tiles. When new inputs are detected, the GRNN provides an output, based on interpolation of past records. This is worked out by calculating the difference between the input vector and the training vector, which gives the probability value of each output neuron. The detection of targets (face images) within an image of a room is performed by once again dividing the room image up into tiles. These tiles make up the input into the trained GRNN. The output of this GRNN determines the probability of the tiles being targets, and those tiles that may be possible targets are cropped out for further examination. The cropped out tiles become the input of the recognition agent.

As a result of the various types of target images that could be possible for the detection agent, no specific physical feature in the images are selected during the training and running of the agent. Rather specific imaging features are target such as number of connected areas present in the image, mean size of the connecting areas, and the standard deviation of the areas.

\section{Experiment and Test Results Using GRNN}

As discussed in the previous section, The Detection Agent is trained using a two layer Generalised Regression Neural Network. The Agent is trained on a particular face image, such as that in Figure 3 (a). After training is complete a new image (Figure 3 (b) that contains similar images to that of Figure 3 (a) embedded within it is presented to the trained detection Agent. It is now the role of the Detection Agent to detect possible regions of interests within Figure 4. These regions of interests would represent regions within Figure 4 where the embedded images may be present. The results obtain from the detection agent (i.e. the images extracted that represent regions of interest from Figure 4) are passed onto the recognition agent to further refine the search and to positively detect and identify the faces. Passing individual images (regions of interest) to the recognition agent may not be very practical as this could possibly greatly hamper the performance of the overall system. A much quicker and 
effective method that could be used as the input to the recognition agent is to input key features extracted from these regions of interest.

The key feature from the region of interest that would be extracted from the Detection Agent are:

- Number of connected areas present in the image,

- Mean size of the connecting areas, and

- The standard deviation of the areas.

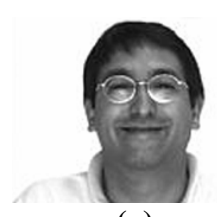

(a)

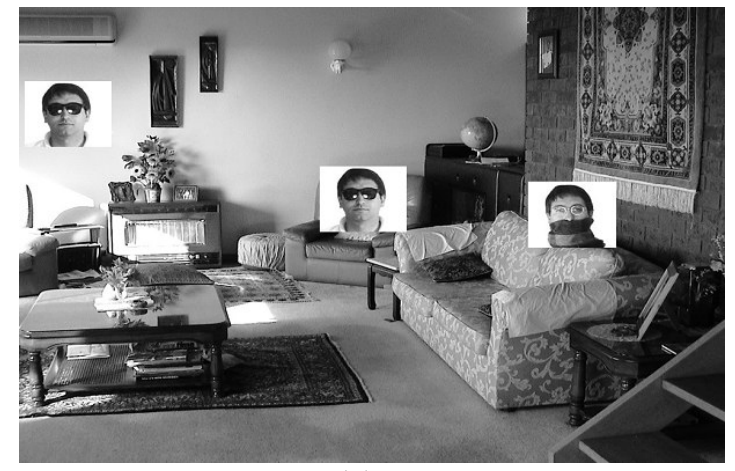

(b)

Fig. 3 (a) Training image of a persons face (b) Test image
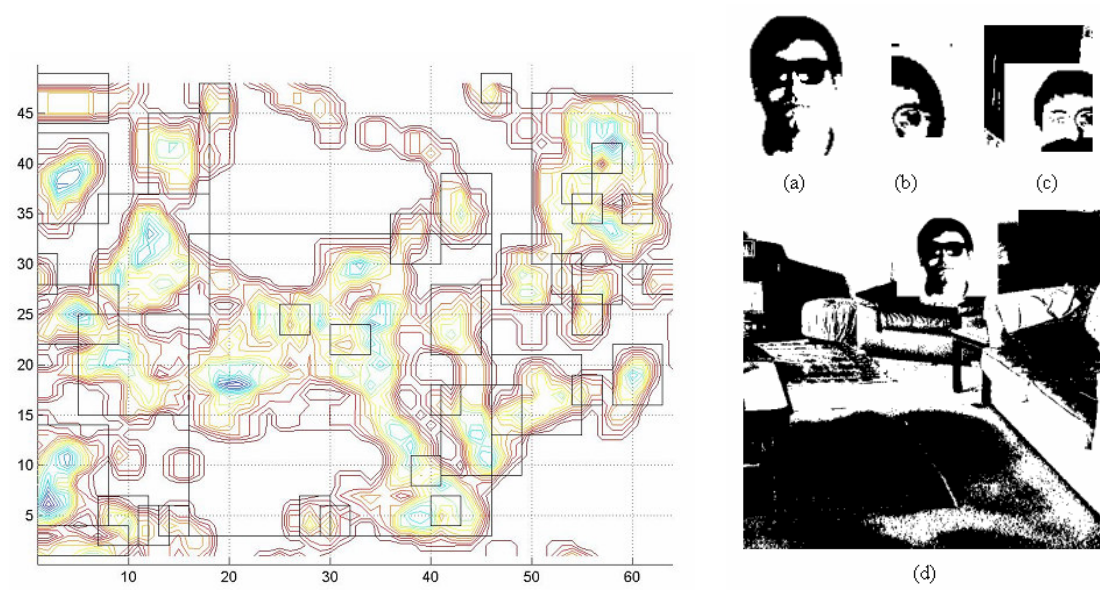

(a)

(b)
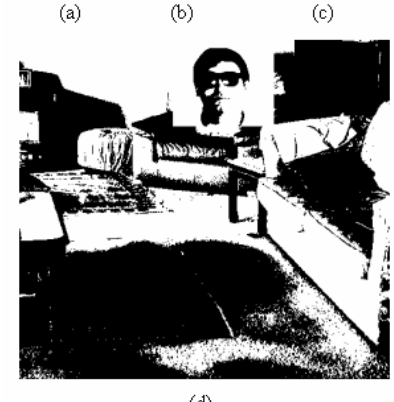

(d)

Fig. 5 (a-d). Extracted image clips

Figure 4 represents the contour density plots of the regions of interest when Figure 3 (b) is passed into the trained detection agent. Each contour density plot refers to an area or a ROI on the test image. Some kind of filtering mechanism is to be used to extract only those contours that may be probable targets. For this purpose a density threshold level is decided and all contour plots above a set density level are extracted. 
The extracted ROI look like the figures illustrated in Figures 5 (a)-(d). These are the clipped images, which acts as the input to the recognition unit. The extracted features such as the number of connected areas present in the image, the mean size of the connecting areas, and the standard deviation of the areas from images shown in Figure 5 are used as the inputs to the recognition agent.

\section{Conclusion}

In this paper a detection agent trained to detect face images was created. An image containing face images was presented to the agent and a test was performed to determine whether any positive hits could be achieved. Initial results from the detection stage of the architecture seems quite encouraging. In the result presented in this paper, forty regions of interest were detected. Amongst the forty, there were four hits. These four are shown in Figure 5. Apart from Figure 5 (a), the other three images either shows too much information or too little information. This problem can be addressed by experimenting with the internal parameters of the Generalised Regression Neural Network.

Even though some of the results show either too much or too little information, the results obtained have shown that all the three embedded images within Figure 3 (b) have been successfully detected. This is a very positive result. A success rate as close as possible to $100 \%$ is required for the success of recognition agent and hence the overall ATR system. If the detection agent fails to detect a possible target, this information would not be passed onto the recognition agent and hence the overall ATR system would fail to positively identify a possible target.

Future work will examine the development of the recognition agent. The recognition agent would perform the same operation as we performed in determining which ROI was a hit and which could be rejected. All forty ROI determined by the detection agent would be passed on to the recognition agent, which would be tasked to identify the positive targets.

\section{References}

[1] Filippidis A., Jain L.C. and Martin N., Fusion of Intelligent Agents for the Detection of Aircraft in SAR Images, IEEE Transactions on Pattern Analysis and Machine Intelligence, Vol. 22, No. 4, 2000.

[2] Joshi V., Jain L.C., Seiffert U., Zyga K., Price R., Leisch F., Neural Techniques in Logo Recognition, First International Workshop on Hybrid Intelligent Systems, Adelaide, Australia, Advances in Soft Computing, Physica-Verlag, pp. 25-32, 2002.

[3] Seiffert U., Logo feature Extraction of Region based properties, Release 1.0, University of Magdeburg, Germany, 2000.

[4] Quirolgico S. and Canfield K., Communicating Neural Network Knowledge between Agents in a Simulated Aerial Reconnaissance System, First International Symposium on Agent Systems and Applications, pp. 242 -254, 1999.

[5] Tecuci G., Boicu M., Marcu D., Stanescu B., Boicu C.and Comello J., Training and Using Disciple Agents: A Case Study in the Military Center of Gravity Analysis Domain, in AI Magazine, AAAI Press, Menlo Park, California, 2002. 
[6] Boicu M., Modeling and Learning with Incomplete Knowledge, $\mathrm{PhD}$ Thesis in Information Technology, Learning Agents Laboratory, School of Information Technology and Engineering, George Mason University, 2002. 